Corresponding author:

Dhamija.Radhika@mayo.edu

(C) 2019 Schultz-Rogers et al. This article is distributed under the terms of the Creative Commons Attribution-NonCommercial License, which permits reuse and redistribution, except for commercial purposes, provided that the original author and source are credited.

Ontology terms: delayed gross motor development; dysphagia; EMG: myopathic abnormalities; episodic generalized hypotonia; lumbar kyphoscoliosis; mildly elevated creatine

phosphokinase; pectus excavatum of inferior sternum; restrictive respiratory insufficiency; speech articulation difficulties; waddling gait

Published by Cold Spring Harbor Laboratory Press

doi:10.1101/mcs.a004309

\section{Novel biallelic variants in MSTO1 associated with mitochondrial myopathy}

\author{
Laura Schultz-Rogers, ${ }^{1}$ Alejandro Ferrer, ${ }^{1}$ Nikita R. Dsouza, ${ }^{2}$ \\ Michael T. Zimmermann, ${ }^{2,3}$ Benn E. Smith, ${ }^{4}$ Eric W. Klee, ${ }^{1,5}$ and Radhika Dhamija ${ }^{6}$ \\ ${ }^{1}$ Center for Individualized Medicine, Mayo Clinic, Rochester, Minnesota 55905, USA; ${ }^{2}$ Bioinformatics Research \\ and Development Laboratory, Genomics Sciences and Precision Medicine Center, Medical College of \\ Wisconsin, Milwaukee, Wisconsin 53226, USA; ${ }^{3}$ Clinical and Translational Sciences Institute, Medical College \\ of Wisconsin, Milwaukee, Wisconsin 53226, USA; ${ }^{4}$ Department of Neurology, Mayo Clinic, Scottsdale, Arizona \\ 85259, USA; ${ }^{5}$ Department of Clinical Genomics, Mayo Clinic, Rochester, Minnesota 55905, USA; ${ }^{6}$ Department \\ of Medical Genetics, Mayo Clinic, Phoenix, Arizona 85054, USA
}

\begin{abstract}
Mitochondrial disorders are caused by nuclear and mitochondrial pathogenic variants leading to defects in mitochondrial function and cellular respiration. Recently, the nuclear-encoded mitochondrial fusion gene MSTO1 (Misato 1) has been implicated in mitochondrial myopathy and ataxia. Here we report on a 30-yr-old man presenting with a maternally inherited NM_018116.3:c.651C>G, p.F217L missense variant as well as a paternally inherited arr[GRCh37] 1q22(155581773_155706887) × 1 deletion encompassing exons 7-14 of MSTO1. His phenotype included muscle weakness, hypotonia, early motor developmental delay, pectus excavatum, and scoliosis. Testing revealed elevated plasma creatine kinase, and electromyogram results were consistent with longstanding generalized myopathy. These phenotypic features overlap well with previously reported patients harboring biallelic MSTO1 variants. Additionally, our patient presents with dysphagia and restrictive lung disease, not previously reported for MSTO1-associated disorders. The majority of patients with disease-associated variants in MSTO1 present with biallelic variants suggesting autosomal recessive inheritance; however, one family has been reported with a single variant and presumed autosomal dominant inheritance. The pattern of inheritance we observed is consistent with the majority of previous reports suggesting an autosomal recessive disorder. We add to our knowledge of the syndrome caused by variants in MSTO1 and provide additional evidence supporting autosomal recessive inheritance. We also describe phenotypic features not reported in previous cases, although further research is needed to confirm they are associated with defects in MSTO1.
\end{abstract}

\section{INTRODUCTION}

Mitochondrial dysfunction leads to a diverse array of disorders most often characterized by myopathic and neurologic symptoms (Chinnery 1993). As the mitochondria are essential for normal aerobic metabolism, tissues and organs with high energy demands such as skeletal muscle are particularly sensitive to mitochondrial disruption (Wallace 1999). Damaging variants in more than 1000 nuclear encoded and mitochondrial genes can affect respiratory chain complexes, mtDNA replication and maintenance, and dynamic fusion and fission processes. Fusion helps maintain a healthy population of mitochondria by distributing mtDNA 
and metabolites and eliminating damaged mitochondria through mitophagy (for review, see El-Hattab et al. 2018).

MSTO1 (Misato 1) is a nuclear gene that contains a region homologous to the Tubulin/ FtsZ, GTPase superfamily, which contains proteins that regulate chromosome segregation in eukaryotes and mitochondrial fission in eukaryotic algae (Kimura and Okano 2007). It encodes a soluble cytoplasmic protein that translocates to the mitochondrial outer membrane and promotes normal fusion dynamics (Kimura and Okano 2007; Gal et al. 2017; Nasca et al. 2017; El-Hattab et al. 2018). In Drosophila, knockdown of DML1, the homolog of human MSTO1, leads to defects in chromosome segregation during mitosis (Miklos et al. 1997). In Saccharomyces cerevisiae, disruption of DML1 causes impaired inheritance of mtDNA and segregation of mitochondria (Gurvitz et al. 2002). Inhibition of MSTO1 in HeLa cells results in nuclear and mitochondrial fragmentation, reduced fusion, and apoptosis, whereas overexpression results in perinuclear aggregations of mitochondria (Kimura and Okano 2007; Gal et al. 2017).

MSTO1 was first associated with human disease in 2017 when Nasca et al. reported on three patients with compound heterozygous variants in MSTO1 presenting with myopathy and cerebellar ataxia (MIM:617675). Functional studies in patient fibroblasts confirmed partially reduced transcript and protein expression as well as fragmentation of the mitochondrial network and decreased mitochondrial fusion (Nasca et al. 2017). To date, a total of 11 patients have been reported in the literature with myopathy and ataxia attributed to pathogenic variants in MSTO1. Consistent phenotypic features observed across patients include onset in the first year of life, severe motor delay, cerebral atrophy on MRI, short stature, myopathic pattern on EMG, and elevated plasma creatine kinase (CK) (Table 1; Nasca et al. 2017; Iwama et al. 2018; Li et al. 2019). Additionally, several patients presented with cognitive deficiencies, skeletal abnormalities, tremors, and retinopathy.

Here we present a 30-yr-old male with phenotypic features consistent with previously reported MSTO1 patients. We report additional phenotypic features seen in our patient including dysphagia and restrictive lung disease, which may be the consequence of the associated myopathy. Our patient has a novel partial gene deletion adding to the spectrum of variants associated with MSTO1-associated mitochondrial myopathy.

\section{RESULTS}

\section{Clinical Presentation}

A 30-yr-old Caucasian male presented with moderate-to-severe proximal and distal muscle weakness and significantly decreased muscle tone. He had congenital hypotonia with poor head control and was diagnosed with "muscular dystrophy of unknown type" at 18 mo of age. His gross and fine motor milestones were significantly delayed, including walking at 26 mo of age and the inability to pedal a bike or run as a child. Learning disability was noted at early childhood. Neurological examination at age 30 was significant for proximal and distal muscle weakness with hypotonia, mild facial weakness with ptosis, diminished muscle bulk, no sensory deficits, and preserved muscle reflexes. Gait was wide based and waddling. Swallowing video fluoroscopy revealed mild oropharyngeal dysphagia. Electromyogram (EMG) results were consistent with a longstanding, generalized myopathy, with electrodiagnostic features that would predict fiber splitting, necrosis, inflammation, or vacuolar change. He has persistently elevated CK levels with titers between 1000 and $2000 \mathrm{U} / \mathrm{L}$. Pulmonary function tests showed a chronic restrictive pattern with forced vital capacity (FVC) at 44\%$49 \%$ of predicted leading to a diagnosis of restrictive lung disease (Fig. 1A,B).

Additional phenotypic features reported in the patient include pectus excavatum that was surgically corrected at age 18, as well as scoliosis characterized by mild reversal of 


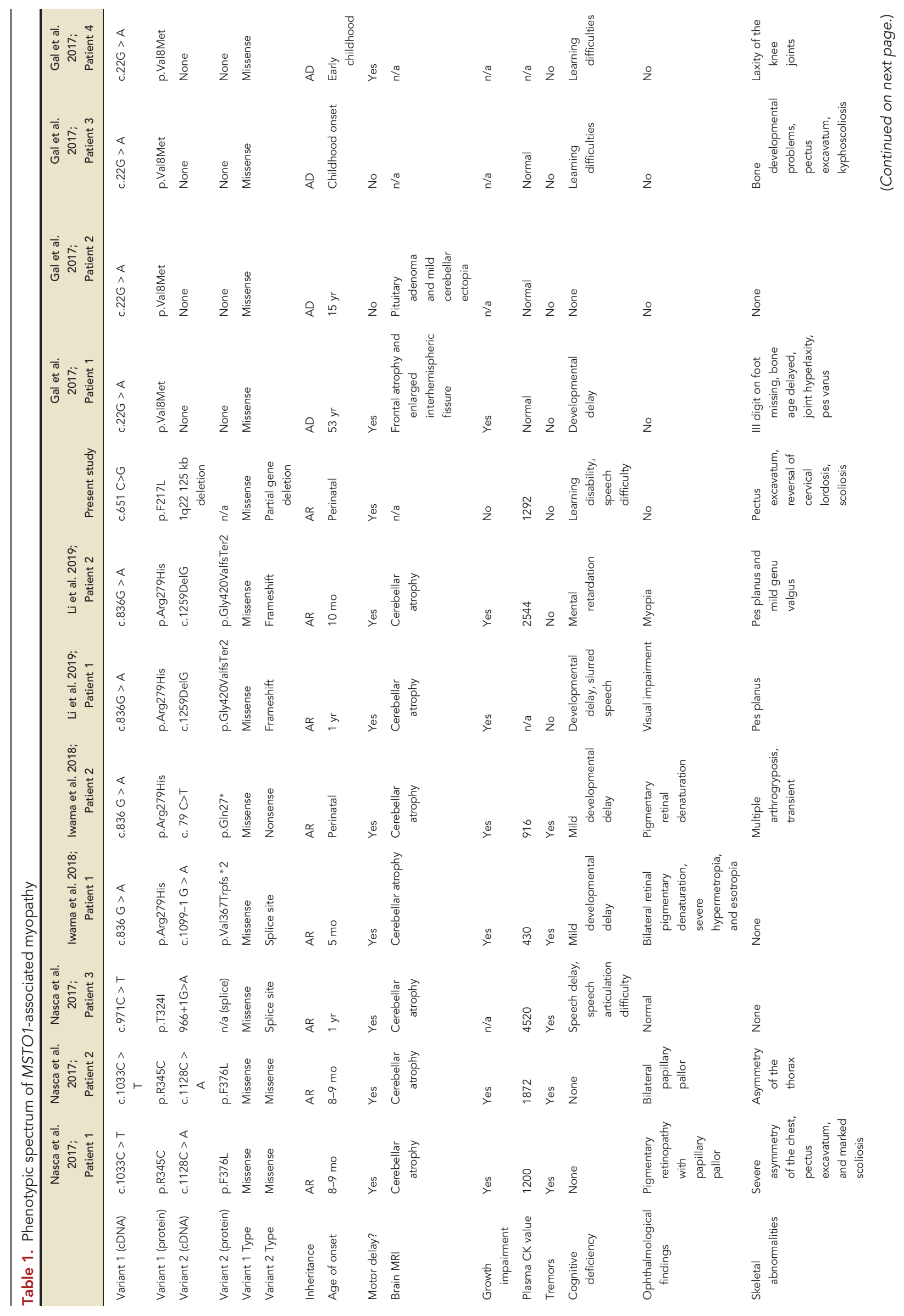




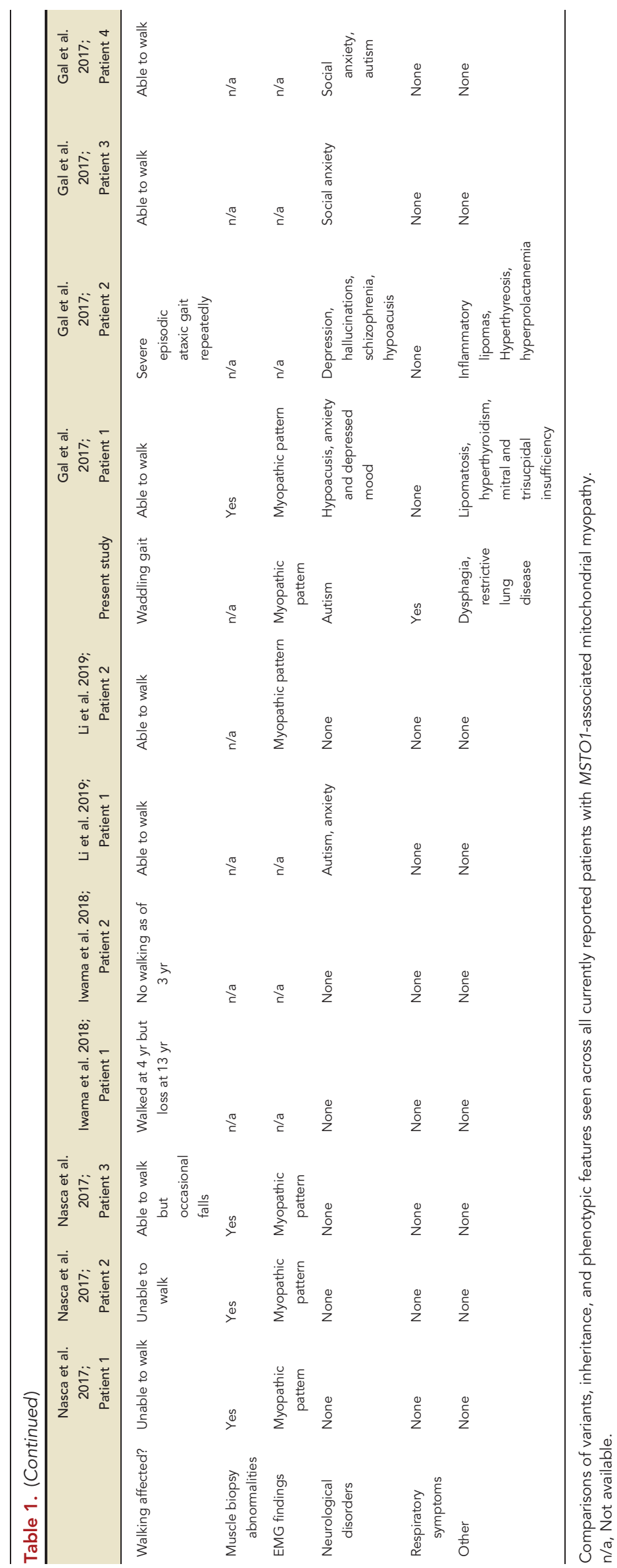




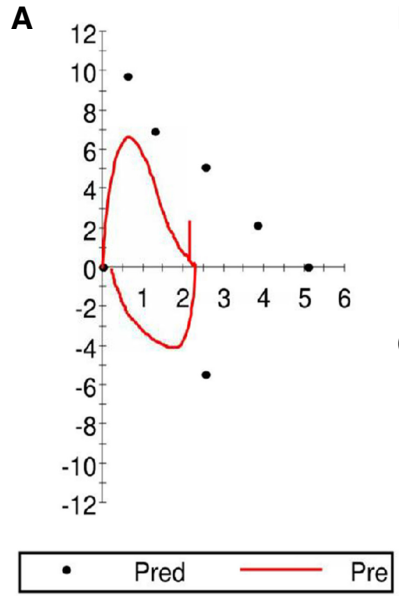

B -- SPIROMETRY --
FVC (L)
FEV 1 (L)
FEV 1/FVC (\%)
FEF 25-75\% (L/sec)
FEF Max (L/sec)
MVV (L/min)
MEP (cmH2O)
MIP (cmH2O)

C

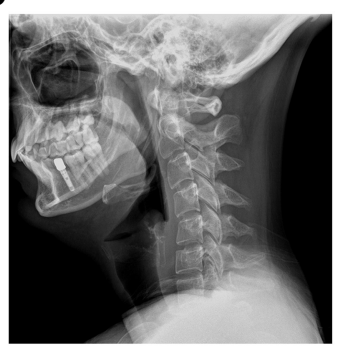

\begin{tabular}{|c|c|c|c|}
\hline $\begin{array}{l}\text { PREI } \\
\text { Pred }\end{array}$ & $\begin{array}{l}\text { ICTED } \\
\text { LLN }\end{array}$ & $\begin{array}{r}\text { CON } \\
\text { Actual } \\
\end{array}$ & \\
\hline 5.09 & 4.25 & $* 2.32$ & $* 45$ \\
\hline 4.16 & 3.47 & $* 2.18$ & $* 52$ \\
\hline 82 & 68 & 94 & 114 \\
\hline 4.24 & 2.33 & 3.83 & 90 \\
\hline 9.74 & 7.30 & $* 6.58$ & $* 67$ \\
\hline $\begin{array}{l}173 \\
173\end{array}$ & 144 & *92 & $* 53$ \\
\hline 231 & 193 & $* 57$ & $* 24$ \\
\hline-125 & -104 & ${ }^{*}-48$ & $* 38$ \\
\hline
\end{tabular}

D

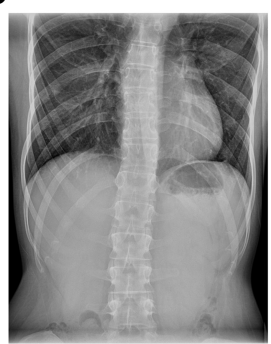

Figure 1. Pulmonary function and skeletal abnormalities in a patient with biallelelic MSTO1 variants. (A) Airflow (L/sec) versus volume (L) displays a severe restriction of pulmonary function. (B) The forced expiratory volume in $1 \mathrm{sec}(\mathrm{FEV} 1) / \mathrm{FVC}$ is normal; the FEV1 is moderately to severely reduced; the FVC is reduced; maximal expiratory pressure (MEP) 24\% predicted, maximal inspiratory pressure (MIP) 38\% predicted. Overall results of the pulmonary function test confirm a chronic restrictive pattern. Skeletal radiographs $(C)$ show mild reversal of cervical lordosis and $(D)$ trace gentle convex curvature of the lumbar spine to the right.

cervical lordosis and a trace gentle convex curvature of the lumbar spine to the right (Fig. 1C, D). An echocardiogram was normal. The family history was unremarkable with the exception of a maternal grandmother who died of complication from ALS at age 74 (Fig. 2).

\section{Genomic Analysis}

Initial genetic testing included a 141-gene panel (Neuromuscular Genetics Panel; Mayo Clinic) and dystrophin deletion/duplication sequencing analysis. None of the variants reported were considered relevant (Table 2). Testing for Pompe disease was also negative. Mitochondrial DNA NGS testing was performed through GeneDx, and a homoplasmic

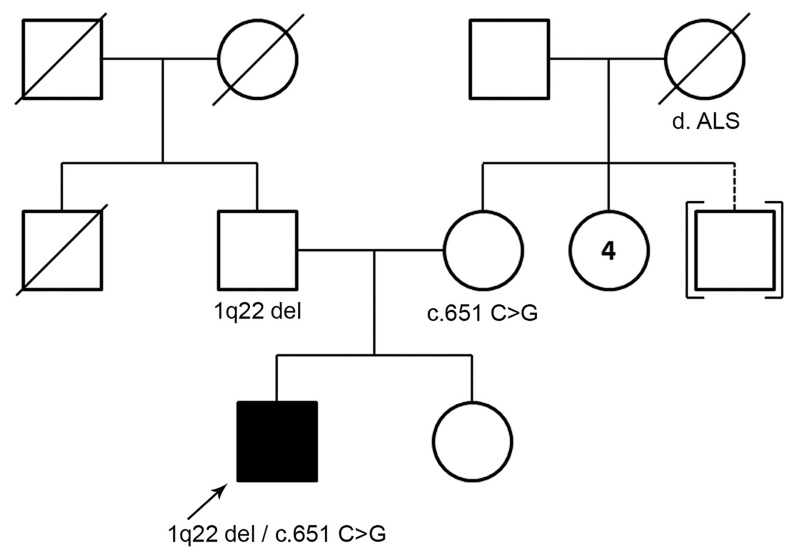

Figure 2. Family pedigree. A three-generation family pedigree in which the proband is the only affected individual. Each MSTO1 variant was inherited from an unaffected parent. 
Table 2. Discarded variants of uncertain significance

\begin{tabular}{|c|c|c|c|c|c|c|c|c|}
\hline Gene & Chromosome & $\begin{array}{l}\text { HGVS DNA } \\
\text { reference }\end{array}$ & $\begin{array}{l}\text { HGVS } \\
\text { protein } \\
\text { reference }\end{array}$ & $\begin{array}{l}\text { Variant } \\
\text { type }\end{array}$ & $\begin{array}{l}\text { Predicted } \\
\text { effect }\end{array}$ & $\begin{array}{c}\text { dbSNP/ } \\
\text { dbVAR ID }\end{array}$ & Genotype & Reason discarded \\
\hline CAVIN1 & $17: 40556955$ & c. $923 A>G$ & p.Y308C & Missense & Substitution & rs146799286 & Heterozygous & $\begin{array}{l}\text { Lack of phenotypic } \\
\text { overlap (no } \\
\text { lipodystrophy); no } \\
\text { second variant found }\end{array}$ \\
\hline SMCHD1 & 18:2732423 & c.3209T>C & p.I1070T & Missense & Substitution & rs113434340 & Heterozygous & $\begin{array}{l}\text { Lack of phenotypic } \\
\text { overlap; no second } \\
\text { variant found in } \\
\text { DUX4 }\end{array}$ \\
\hline TMEM43 & 3:14177385 & c.859C $>\mathrm{T}$ & p.H287Y & Missense & Substitution & rs780299346 & Heterozygous & $\begin{array}{l}\text { Lack of phenotypic } \\
\text { overlap (no } \\
\text { cardiovascular } \\
\text { symptoms) }\end{array}$ \\
\hline TTN & 2:179419690 & c. $80792 \mathrm{~T}>\mathrm{G}$ & p.L26931R & Missense & Substitution & rs72648234 & Heterozygous & $\begin{array}{l}\text { Autosomal dominant } \\
\text { association with } \\
\text { disease but } \\
\text { previously reported } \\
\text { in unaffected } \\
\text { individuals }\end{array}$ \\
\hline TTN & 2:179587094 & c. $18688 \mathrm{G}>\mathrm{A}$ & p.A6230T & Missense & Substitution & rs759713604 & Heterozygous & $\begin{array}{l}\text { Autosomal dominant } \\
\text { association with a } \\
\text { disease but } \\
\text { previously reported } \\
\text { in unaffected } \\
\text { individuals }\end{array}$ \\
\hline
\end{tabular}

Prior to whole-exome sequencing, a 141-gene Neuromuscular Genetics Panel was performed on patient DNA. Several variants of uncertain significance were reported, but all were discarded as candidates for the reasons described in the table.

variant of uncertain significance (m.8045A>G, p.1154V) was reported in MT-CO2. This variant was also considered not diagnostic as the patient's unaffected mother was homoplasmic for the same variant. Whole-exome sequencing performed through GeneDx reported two potentially clinically relevant variants; a maternally inherited NM_018116.3:c.651C>G, p.F217L missense variant of uncertain significance and a likely pathogenic paternally inherited arr[GRCh37] 1q22(155581773_155706887) × 1 copy-number change (Table 3; Fig. 3A). The copy-number change was originally detected through GeneDx's NGS-CNV caller (Retterer et al. 2015) and was confirmed by GeneDx with the GenomeDx v5 chromosomal array. The missense variant was confirmed by GeneDx via direct gene-specific PCR.

The p.F217L missense variant lies in exon 7 of 14 in the tubulin domain. The affected amino acid is highly conserved across species (Fig. 3B), and in silico predictions agree the

\begin{tabular}{|c|c|c|c|c|c|c|c|c|}
\hline Gene & Chromosome & $\begin{array}{l}\text { HGVS DNA } \\
\text { reference }\end{array}$ & $\begin{array}{l}\text { HGVS protein } \\
\text { reference }\end{array}$ & $\begin{array}{l}\text { Variant } \\
\text { type }\end{array}$ & $\begin{array}{l}\text { Predicted } \\
\text { effect }\end{array}$ & $\begin{array}{c}\text { dbSNP/ } \\
\text { dbVAR ID }\end{array}$ & Genotype & $\begin{array}{l}\text { Parent of } \\
\text { origin }\end{array}$ \\
\hline MSTO1 & 1:155581864 & c. $651 C>G$ & p.Phe217Leu & Missense & Substitution & rs776826330 & Heterozygous & Mother \\
\hline MSTO1 & $1 q 22$ & $\begin{array}{c}1 q 22(155581773 \\
155706887) \times 1\end{array}$ & $\mathrm{n} / \mathrm{a}$ & $\begin{array}{l}\text { Copy- } \\
\text { number } \\
\text { change }\end{array}$ & $\begin{array}{c}\text { Partial gene } \\
\text { deletion }\end{array}$ & $\mathrm{n} / \mathrm{a}$ & Heterozygous & Father \\
\hline
\end{tabular}




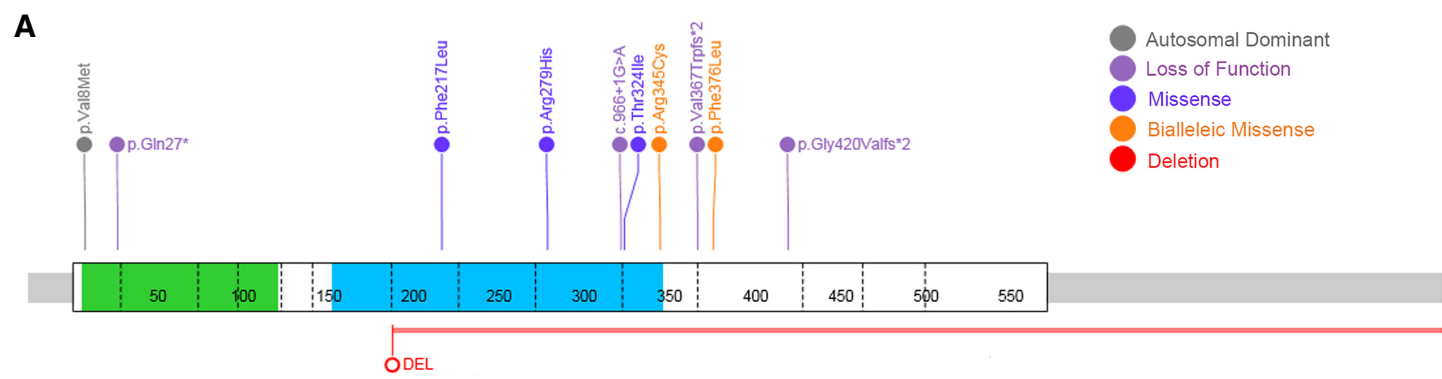

B

\begin{tabular}{|c|c|c|c|c|c|c|c|c|c|c|c|}
\hline Human & E & D & $\mathbf{R}$ & $\mathbf{L}$ & H & $\mathbf{F}$ & $\mathbf{Y}$ & v & E & E & C \\
\hline Chimp & E & D & $\mathbf{R}$ & L & $\mathbf{H}$ & $\mathbf{F}$ & Y & v & E & E & C \\
\hline \multicolumn{12}{|l|}{ Northern white-cheeked gibbon } \\
\hline Macaque & $\mathbf{E}$ & D & $\mathbf{R}$ & $\mathbf{L}$ & H & $\mathbf{F}$ & $\mathbf{Y}$ & v & E & $\mathbf{E}$ & C \\
\hline Rat & $\mathbf{E}$ & D & $\mathbf{R}$ & $\mathbf{L}$ & H & $\mathbf{F}$ & $Y$ & v & $\mathbf{E}$ & E & C \\
\hline Mouse & $\mathbf{E}$ & D & $\mathbf{R}$ & $\mathbf{L}$ & H & $\mathbf{F}$ & $Y$ & v & E & E & C \\
\hline Dog & $\mathbf{E}$ & D & $\mathbf{R}$ & $\mathbf{L}$ & H & $\mathbf{F}$ & $Y$ & $\mathbf{L}$ & E & E & C \\
\hline Chicken & E & D & $\mathbf{R}$ & $\mathbf{L}$ & H & $\mathbf{F}$ & $Y$ & A & E & E & C \\
\hline Frog & E & $\mathbf{E}$ & $E$ & L & G & $Y$ & L & $\mathbf{L}$ & $\mathbf{P}$ & I & V \\
\hline Tetraodon & E & D & K & $\mathbf{L}$ & H & $\mathbf{F}$ & $\mathbf{F}$ & A & E & $E$ & C \\
\hline Zebrafish & E & D & $\mathbf{R}$ & L & H & $\mathbf{F}$ & $\mathbf{F}$ & I & E & E & C \\
\hline Fruitfly & C & D & $\mathbf{R}$ & I & $\mathbf{R}$ & L & $Y$ & v & E & E & C \\
\hline
\end{tabular}

Figure 3. Gene location of disease-associated MSTO1 variants. (A) Map of currently known variants associated with mitochondrial myopathy. Green and blue regions denote the tubulin domains. Most of the variants cluster in the known tubulin domains. Figure generated using ProteinPaint, St. Jude Children's Research Hospital. (B) The amino acid affected by our patient's missense variant (red box) shows high conservation among orthologs.

variant is likely damaging (PolyPhen-2 [Adzhubei et al. 2010], SIFT [Sim et al. 2012], M-CAP [Jagadeesh et al. 2016], and PredictSNP2 [Bendl et al. 2016]). The p.F217L variant could possibly affect the structural conformation as it is a part of an $a$-helix and the only hydrophobic residue within a region of charged and hydrophilic residues. The 1q22 (155581773_155706887 × 1) copy-number variant is a 125-kb deletion encompassing exons 7-14 of MSTO1, exon 7-13 of the MSTO2P pseudogene, and all of DAP3 (death-associated protein 3) and YY1AP1 (YY1 associated protein 1). Although the homology between MSTO1 and its pseudogene could affect the detection of CNVs in this gene, the fact that the missense variant in exon 7 was called as homozygous supports the presence of a deletion in this gene. MSTO2P is not associated with any human disorders other than potential links to cancer, which are not reported in our patient. Biallelic loss of function variants in YY1AP1 are associated with Grange syndrome, whereas missense and small insertion variants in DAP3 have been associated with metabolic disorders and autism spectrum disorder. Because our patient does not have a second damaging variant reported in these genes and his phenotype does not overlap with these syndromes, we concluded that it is not likely that a single-copy loss of any of these three genes is contributing to our patient's phenotype.

MSTO1 is not predicted to be intolerant to loss of function or missense variation based on gene constraints in gnomAD ( $\mathrm{pLI}=0, Z=0$ ) (Lek et al. 2016). However, as autosomal recessive inheritance is expected, it is possible to see damaging variants in a heterozygous state in the normal population. The p.F217L variant is observed at a very low frequency in gnomAD (0.007\%) with no homozygotes reported. This variant has not been previously reported in HGMD or ClinVar. The 1q22(155581773_155706887) deletion has not been previously observed in normal or diseased individuals. Based on the Database of Genomic Variants, similarly sized deletions have been detected in heterozygotes in the normal population (nsv436522, nsv3043, nsv48213) (MacDonald et al. 2014). 


\section{DISCUSSION}

Here we present a patient with novel compound heterozygous variants in MSTO1 and mitochondrial myopathy. His phenotypic presentation overlaps well with previously reported patients including early-onset muscle weakness, motor delay, elevated plasma CK, myopathic pattern on EMG, and skeletal abnormalities. At 30 years old, our patient is the oldest reported patient with biallelic variants in MSTO1, adding important knowledge about potential life span for other similarly affected patients. We report features including restrictive lung disease and dysphagia not previously identified in patients with MSTO1-associated mitochondrial myopathy. These additional clinical findings may be the consequence of the associated myopathy. Awareness of the full phenotypic spectrum associated with this disorder is critical for proper diagnosis of future patients. As the role of tubulins in mitochondrial function becomes more apparent (Mado et al. 2019), it will be important to elucidate the role of MSTO1 in normal mitochondrial dynamics and how disruption leads to myopathy as well as skeletal, retinal, and other phenotypic features observed.

The p.F217L missense variant seen in our patient lies in the second tubulin domain on the MSTO1 protein. Five of the six previously reported pathogenic missense variants lie in one of the tubulin domains, suggesting these regions are critical for normal protein function and/or stability. Regions of proteins that bind to other proteins often have a hydrophobic center with a hydrophilic and charged ring surrounding them, much like the region around p.F217. If protein interactions with the MSTO1 tubulin domain occur at p.F217, then p.L217 may be compatible with the overall pattern of protein interfaces, but with a reduced and smaller hydrophobic center, which may not be as specific for its binding partners.

Similar to our patient, five of the 11 previously reported patients were compound heterozygotes with one missense variant and one loss-of-function variant (Fig. 3A). The two most severely affected patients were compound heterozygotes for two missense variants. Nasca et al. (2017) hypothesized that the reduction in protein expression in these patients was due to instability caused by the missense variants. In the gnomAD database of healthy individuals, there is only one individual reported as homozygous for any loss of function allele and this call was flagged as dubious. This suggests that MSTO1 is associated with disease in an autosomal recessive manner wherein biallelic loss of function variants are likely lethal but retention of a partially functional missense allele in conjunction with a loss of function allele results in a disease state. This is supported by studies in Drosophila in which null mutations are embryonic lethal (Miklos et al. 1997). Here we present an additional patient with a loss-of-function variant, in this case a partial gene deletion, in combination with a missense variant in MSTO1 resulting in mitochondrial myopathy.

Contrary to the other reported cases, Gal et al. reported a heterozygous missense variant in members of a family presenting with myopathy, ataxia, and neurodevelopmental impairments (Gal et al. 2017). A second alteration in MSTO1 was not detected with Sanger sequencing of the genomic DNA or real-time PCR analysis of copy-number variation. The authors proposed autosomal dominant inheritance of a multisystemic clinical phenotype. However, the phenotypic features in this family do not overlap well with other reported patients (Table 1). In particular, the age of onset was later (15-53 yr old), plasma CK levels were normal, and cerebellar atrophy was not observed. Functional studies in fibroblasts from patients 1 and 2 (as labeled in the Gal et al. study) did confirm a decrease in transcript and protein expression, mitochondrial fusion, and fragmented and aggregated mitochondria. These discrepant reports may be explained by a genotype/phenotype correlation in which the mode of inheritance depends on the location of the variant or a separate underlying genetic condition.

This case adds to our current knowledge on mitochondrial myopathy associated with biallelic pathogenic variants in MSTO1. We present novel phenotypic features not previously 
COLD SPRING HARBOR Molecular Case Studies
Novel biallelic variants in MSTO1 myopathy described in association with MSTO1-related disease and add support to reports of autosomal recessive inheritance. We expect that additional MSTO1 cases and further research will elucidate the full clinical spectrum of this disorder.

\section{METHODS}

Whole-exome sequencing was performed by GeneDx. The mean depth of coverage was $157 \times$ and the quality threshold (percentage of sequence covered by at least 10 reads) was $98.9 \%$. Briefly, a proprietary capture system was used for next-generation sequencing and targets were sequenced with paired-end reads on an Illumina platform. Reads were aligned to GRCH37/UCSC hg 19. Sequence variants and deletions or duplications involving three or more coding exons were called with XomeAnalyzer. Variants were reported according to the Human Genome Variation Society (HGVS) guidelines. Missense variants were clinically confirmed with direct gene-specific PCR. Copy-number variants were clinically confirmed by chromosomal microarray. Briefly, the GeneDx GenomeDx v5 chromosomal array contains 118,000 oligonucleotide probes and 66,000 SNP probes throughout the genome used for detecting copy-number variants.

The sequencing coverage was as follows: mean depth of coverage was $157 \times$ and the quality threshold was $98.9 \%$.

As a separate test from whole-exome sequencing, mitochondrial sequencing and deletion testing of the mitochondrial genome were performed by GeneDx. Briefly, the mitochondrial genome was amplified and sequenced using a solid state sequencing-by-synthesis process and the sequence was compared to the revised Cambridge Reference Sequence (rCRS). Disease-associated variants were confirmed by conventional dideoxy sequence analysis.

\section{ADDITIONAL INFORMATION}

\section{Data Deposition and Access}

Single-nucleotide variants identified and interpreted by GeneDx can be found in the ClinVar database (https://www.ncbi.nih.nlm.gov/clinvar/) under accession number SCV000965685. Patient permission was not granted for raw sequencing data deposition.

\section{Ethics Statement}

This study was approved by the Mayo Clinic institutional review board and all participants provided written informed consent for genetic testing.

\section{Acknowledgments}

We would like to thank the patient and his family for participating in this study.

\section{Author Contributions}

L.S.-R., A.F., and E.W.K. analyzed patient variants and wrote the manuscript. R.D. and B.E.S. oversaw patient care and genetic interpretation. N.R.D. and M.T.Z. interpreted protein structure. All authors reviewed and approved the final manuscript.

\section{Funding}

This study was supported by the Mayo Clinic Center for Individualized Medicine. 


\section{REFERENCES}

Adzhubei IA, Schmidt S, Peshkin L, Ramensky VE, Gerasimova A, Bork P, Kondrashov AS, Sunyaev SR. 2010. A method and server for predicting damaging missense mutations. Nat Methods 7: 248-249. doi:10.1038/ nmeth0410-248

Bendl J, Musil M, Štourač J, Zendulka J, Damborský J, Brezovský J. 2016. PredictSNP2: a unified platform for accurately evaluating SNP effects by exploiting the different characteristics of variants in distinct genomic regions. PLoS Comput Biol 12: e1004962. doi:10.1371/journal.pcbi.1004962

Chinnery PF. 1993. Mitochondrial disorders overview. In GeneReviews ${ }^{\circledR}$ (ed. Adam MP, et al.). University of Washington, Seattle.

El-Hattab AW, Suleiman J, Almannai M, Scaglia F. 2018. Mitochondrial dynamics: biological roles, molecular machinery, and related diseases. Mol Genet Metab 125: 315-321. doi:10.1016/j.ymgme.2018.10.003

Gal A, Balicza P, Weaver D, Naghdi S, Joseph SK, Várnai P, Gyuris T, Horváth A, Nagy L, Seifert EL, et al. 2017. MSTO1 is a cytoplasmic pro-mitochondrial fusion protein, whose mutation induces myopathy and ataxia in humans. EMBO Mol Med 9: 967-984. doi:10.15252/emmm.201607058

Gurvitz A, Hartig A, Ruis H, Hamilton B, de Couet HG. 2002. Preliminary characterisation of DML1, an essential Saccharomyces cerevisiae gene related to misato of Drosophila melanogaster. FEMS Yeast Res 2: 123135.

Iwama K, Takaori T, Fukushima A, Tohyama J, Ishiyama A, Ohba C, Mitsuhashi S, Miyatake S, Takata A, Miyake $\mathrm{N}$, et al. 2018. Novel recessive mutations in MSTO1 cause cerebellar atrophy with pigmentary retinopathy. J Hum Genet 63: 263-270. doi:10.1038/s10038-017-0405-8

Jagadeesh KA, Wenger AM, Berger MJ, Guturu H, Stenson PD, Cooper DN, Bernstein JA, Bejerano G. 2016. M-CAP eliminates a majority of variants of uncertain significance in clinical exomes at high sensitivity. Nat Genet 48: 1581-1586. doi:10.1038/ng.3703

Kimura M, Okano Y. 2007. Human Misato regulates mitochondrial distribution and morphology. Exp Cell Res 313: 1393-1404. doi:10.1016/j.yexcr.2007.02.004

Lek M, Karczewski KJ, Minikel EV, Samocha KE, Banks E, Fennell T, O’Donnell-Luria AH, Ware JS, Hill AJ, Cummings BB, et al. 2016. Analysis of protein-coding genetic variation in 60,706 humans. Nature 536: 285-291. doi:10.1038/nature19057

Li K, Jin R, Wu X. 2019. Whole-exome sequencing identifies rare compound heterozygous mutations in the MSTO1 gene associated with cerebellar ataxia and myopathy. Eur J Med Genet doi:10.1016/j.ejmg .2019.01.013

MacDonald JR, Ziman R, Yuen RK, Feuk L, Scherer SW. 2014. The Database of Genomic Variants: a curated collection of structural variation in the human genome. Nucleic Acids Res 42: D986-D992. doi:10.1093/ nar/gkt958

Mado K, Chekulayev V, Shevchuk I, Puurand M, Tepp K, Kaambre T. 2019. On the role of tubulin, plectin, desmin and vimentin in the regulation of mitochondrial energy fluxes in muscle cells. Am J Physiol Cell Physiol 316: C657-C667. doi:10.1152/ajpcell.00303.2018

Miklos GL, Yamamoto M, Burns RG, Maleszka R. 1997. An essential cell division gene of Drosophila, absent from Saccharomyces, encodes an unusual protein with tubulin-like and myosin-like peptide motifs. Proc Natl Acad Sci 94: 5189-5194. doi:10.1073/pnas.94.10.5189

Nasca A, Scotton C, Zaharieva I, Neri M, Selvatici R, Magnusson OT, Gal A, Weaver D, Rossi R, Armaroli A, et al. 2017. Recessive mutations in MSTO1 cause mitochondrial dynamics impairment, leading to myopathy and ataxia. Hum Mutat 38: 970-977. doi:10.1002/humu.23262

Retterer K, Scuffins J, Schmidt D, Lewis R, Pineda-Alvarez D, Stafford A, Schmidt L, Warren S, Gibellini F, Kondakova A, et al. 2015. Assessing copy number from exome sequencing and exome array CGH based on CNV spectrum in a large clinical cohort. Genet Med 17: 623-629. doi:10.1038/gim.2014.160

Sim NL, Kumar P, Hu J, Henikoff S, Schneider G, Ng PC. 2012. SIFT web server: predicting effects of amino acid substitutions on proteins. Nucleic Acids Res 40: W452-W457. doi:10.1093/nar/gks539

Wallace DC. 1999. Mitochondrial diseases in man and mouse. Science 283: 1482-1488. doi:10.1126/science .283 .5407 .1482 


\section{COLD SPRING HARBOR Molecular Case Studies}

\section{Novel biallelic variants in MSTO1 associated with mitochondrial myopathy}

Laura Schultz-Rogers, Alejandro Ferrer, Nikita R. Dsouza, et al.

Cold Spring Harb Mol Case Stud 2019, 5: a004309 originally published online October 11, 2019 Access the most recent version at doi: $10.1101 / \mathrm{mcs} .0004309$

References This article cites 17 articles, 3 of which can be accessed free at: http://molecularcasestudies.cshlp.org/content/5/6/a004309.full.html\#ref-list-1

License This article is distributed under the terms of the Creative Commons Attribution-NonCommercial License, which permits reuse and redistribution, except for commercial purposes, provided that the original author and source are credited.

Email Alerting Receive free email alerts when new articles cite this article - sign up in the box at the Service top right corner of the article or click here. 